\title{
Application of Earth Observation Data and Standardized Precipitation Index Based Approach for Meteorological Drought Monitoring, Assessment and Prediction Over Kutch, Gujarat, India
}

\author{
Sumanta Das ${ }^{1 *}$, Malini Roy Choudhury ${ }^{1}$, Sujay Gandhi ${ }^{1}$, Viral Joshi ${ }^{1}$ \\ ${ }^{1}$ Dept. of Civil Engg., Smt. S.R.Patel Engg.College (Affiliated with Gujarat Technological University), Mehsana \\ 384170, GUJARAT-IN
}

Corresponding author*

E-mail: sumanvu_27@yahoo.co.in

Received 19 June 2016

Accepted 30 June 2016

\begin{abstract}
Drought is a natural phenomenon which differs from other natural hazards by its slow accumulating process and its indefinite commencement and termination. The present study addresses water deficiency and drought occurrence over Kutch district, Gujarat, because nearly $45 \%$ of the whole Kutch district is severely suffering by deficiency of water. Earth observation data (LANDSAT ETM+) and Standardized Precipitation Index were used to analyze drought severity. Daily rainfall data over the study area were obtained from Indian Meteorological Department (IMD) for the period of study (1990-2014) and geo-referenced for further analyses. Using Remote Sensing and GIS techniques, rainfall variability map over the period of study has been prepared to show rainfall distribution and land use and land cover map is prepared to show the area under different land use classes and impacts of drought over land uses. Standardized Precipitation Index (SPI) was generated for each block wise and scenario of drought development has been analyzed using decadal data set for the study period (1990-2014). The present study suggests method and techniques for continuous drought monitoring by linking temporal earth observation and rainfall data. The methodology will be very useful for the development of a regional drought monitoring system.
\end{abstract}

Keywords: Drought, SPI, drought severity, Rainfall variability, Remote Sensing

\section{Introduction}

Drought has long been recognized as one of the major causes of human misery and being the natural disaster that annually claims the most victims. Droughts are recurring climatic events, which often hit South Asia, bringing significant water shortages, economic losses and adverse social consequences. Water scarcity can be said as the cause and effect of drought. Drought occurs whenever and wherever the links in the hydrological cycle is broken or is destabilized. Drought is a slow poison, no one knows when it creeps in, it can last any number of days and its severity cannot be predicted.

It has extensive spatial dimension and thus can have serious implications on the socioeconomic stability of an entire region. It is not possible to avoid droughts. But drought preparedness can be developed and drought impacts can be managed. The success of both depends, amongst the others, on how well the droughts are defined and drought characteristics quantified (Smakhtin V. U., Hughes D. A., 2004). Hence, preparedness to drought should form the important part of national environmental policies. The levels of drought preparedness in countries of the region differ significantly. A drought is a complex phenomenon that can be defined from several perspectives (Wilhite D., Glantz M. R., 1987). Drought is a normal feature of climate and its recurrence is inevitable (Mishra A. K., Desai V. R., 2005). Most often from literatures, operational definitions identify the beginning, end, spatial extent and severity of a drought. They are often region-specific and are based on scientific reasoning, which follows the analysis of certain amounts of hydro meteorological information. They are beneficial in developing drought policies, monitoring systems, mitigation strategies and preparedness plans. 
A Meteorological drought is usually on the basis of the degree of dryness (in comparison to some "normal" or average amount) and the duration of the dry period (Palmer W. C., 1965). Definitions of meteorological drought must be considered as region specific since the atmospheric conditions that result in deficiencies of precipitation are highly variable from region to region. Standard Precipitation Index (SPI) is more reliable for detecting emerging drought and it is becoming an increasingly important tool for assessing moisture condition and initiating drought response actions at state, regional and local level; planning for drought; monitoring drought; drought risk and impact analysis; and mitigating drought by putting a drought plan together for water conservation (Rawat K.S., Tripathi V.K., George Paul., Mishra A.K., 2014; Wilhite D. A., Hayes M. J., Svoboda M. D., 2000). In present study an attempt has been made to evaluate the drought of Agro climate zones of India by SPI. This paper highlights the application of the SPI as a measure for drought monitoring in the different agro-climatic zones of India.

Earth observation data and Geospatial techniques can assist for detection and mapping of the agricultural drought prone areas including monitoring appropriate site for specific mitigation actions (Das S., Choudhury MR, Nanda S., 2013). Thus, SPI based drought risk zonation will be produced from this study, can be useful for policy makers to take appropriate actions depending upon the risk level. On the other hand it is helpful for researchers to generate the information including selection of drought prone area as well crop management and soil moisture conservation practices. Moreover, it may be helpful for development agencies and Government Organization (NGO) for drought management.

This paper addresses a serious issue due to scarcity of rainfall and drought occurrence catastrophically over the Kutch district, Gujarat. The first and foremost objective of the study is to assess the spatiotemporal occurrence of meteorological drought and their impacts by satellite Remote Sensing techniques and SPI analysis and suggest a method and techniques for continuous drought monitoring by linking temporal earth observation and rainfall data.

\section{Materials and Methods}

\section{Study Area}

Kutch district (also spelled as Kachchh) is a district of Gujarat state in western India. Covering an area of $45,652 \mathrm{~km}^{2}$ (Figure 1), it is the largest district of India. Kutch literally means something which intermittently becomes wet and dry; a large part of this district is known as Kutch which is shallow wetland which submerges in water during the rainy season and becomes dry during other seasons.

The Rann is famous for its marshy salt flats which become snow white after the shallow water dries up each season before the monsoon rains. Kutch District, at 45,692 square kilometres $(17,642 \mathrm{sq}$ mile), is the largest district in India. The administrative headquarters is in Bhuj which is geographically in the centre of district. Other main towns are Gandhidham, Rapar, Nakhatrana, Anjar, Mandvi, Madhapar, Mundra and Bhachau. Kutch has 969 villages. Kala Dungar (Black Hill) is the highest point in Kutch at 458 meters $(1,503 \mathrm{ft})$.

\section{Data}

\section{Spatial Data:}

1. Satellite Data (LANDSAT ETM+, 2014)

2. Survey of India Topographical sheet $(1: 50,000)$

\section{Non-spatial data:}

\section{Software used:}

1. Rainfall data (1990-2014)

1. ArcGIS 9.3

2. ERDAS Imagine 9.2

3. MS Office 


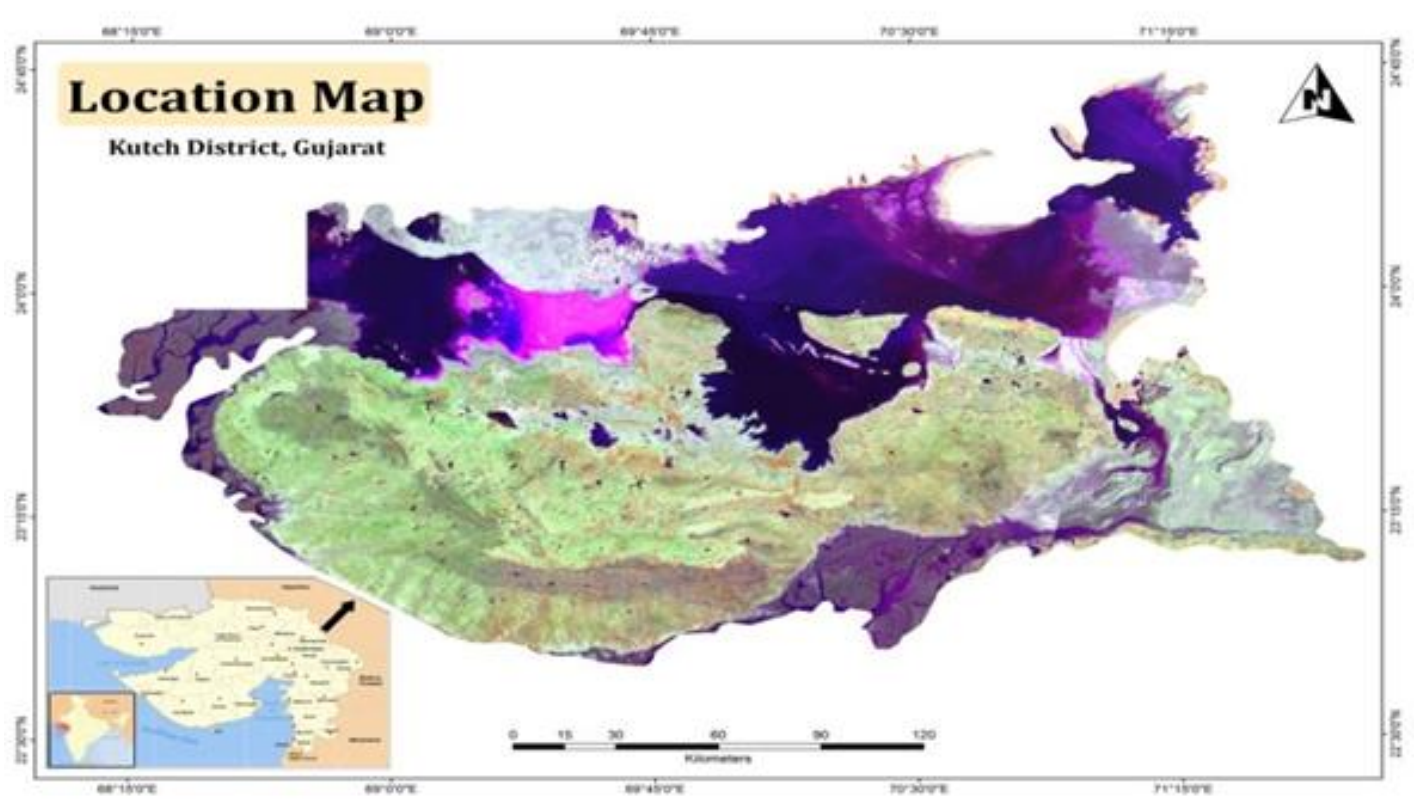

Figure 1. Location Map

\section{Procurement and Analysis of Rainfall Data}

Daily satellite derived precipitation data were acquired from the IMD. The need for satelliteestimated precipitation arises due to the poorly spatially distributed ground rainfall data. The daily rainfall estimation files have been provided for the Kutch beginning from Jan 01, 1990. The product is updated three times daily at around $9 \mathrm{am}, 1 \mathrm{pm}$, and $9 \mathrm{pm}$ eastern local time and covers a 24-hour period of accumulated precipitation. The gauge-based analysis of daily precipitation is derived by interpolating reports from 280 GTS stations over the target domain. The GPI estimates are based on up to 48 times of half-hourly IR observations but they are indirect in nature and exhibit significant bias over land. The SSM/I and the AMSU-B estimates are more physically based but they have incomplete spatial coverage and large random error due to the limited sampling. In general, all of the individual data sources show similar large-scale distribution patterns but present differences in smaller scale features and in magnitude. Three major deficiencies exist in these individual data sources, i.e., incomplete spatial coverage; significant random error; and non-negligible bias.
Four kinds of observation-based data sets were used as inputs to construct the merged analysis of daily precipitation (Figure 2). The figure shows an example of the input daily precipitation fields for Jan, 1990. The merged analysis presents similar spatial distribution patterns with those of satellite estimates while its magnitude is close to the gauge-based analysis over areas with gauge data.

The rainfall estimates were compared with the station recorded precipitation values. Statistics were generated upon completion of all processes and the results are shown in Table 1.GTS, GPI, SSM/I, and AMSU-A data are considered the operational inputs of the RFE 2.0 algorithm. It can be seen that this set of inputs produces the highest correlation between estimated precipitation and actual station rainfall amounts, with a relatively small bias.

\section{Standardized Precipitation Index (SPI)}

Standard Precipitation Index is a state-of-the-art method developed for assessing climatic variability (McKee T. B., Doesken N. J., Kleist J.,1995; McKee T. B., Doesken N. J., Kleist J., 1993). The Standardized Precipitation Index (SPI) is a tool which was developed primarily for defining and monitoring drought. It allows 
an analyst to determine the rarity of a drought at a given time scale (temporal resolution) of interest for any rainfall station with historic data. It can also be used to determine periods of anomalously wet events. The purpose is to assign a single numeric value to the precipitation that can be compared across regions with markedly different climates. Technically, the SPI is the number of standard deviations that the observed value would
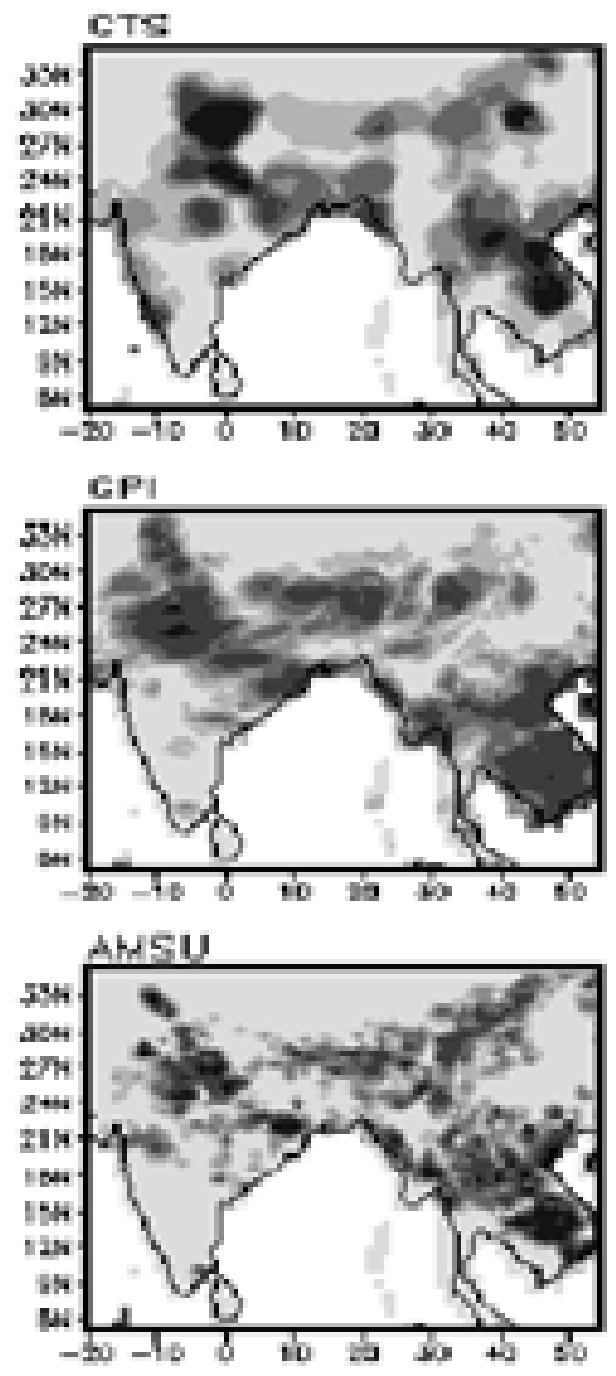

deviate from the long-term mean, for a normally distributed random variable. Since precipitation is not normally distributed, a transformation is first applied so that the transformed precipitation values follow a normal distribution. The SPI was designed to quantify the precipitation deficit for multiple time scales. These time scales reflect the impact of drought on the availability of the different water resources.
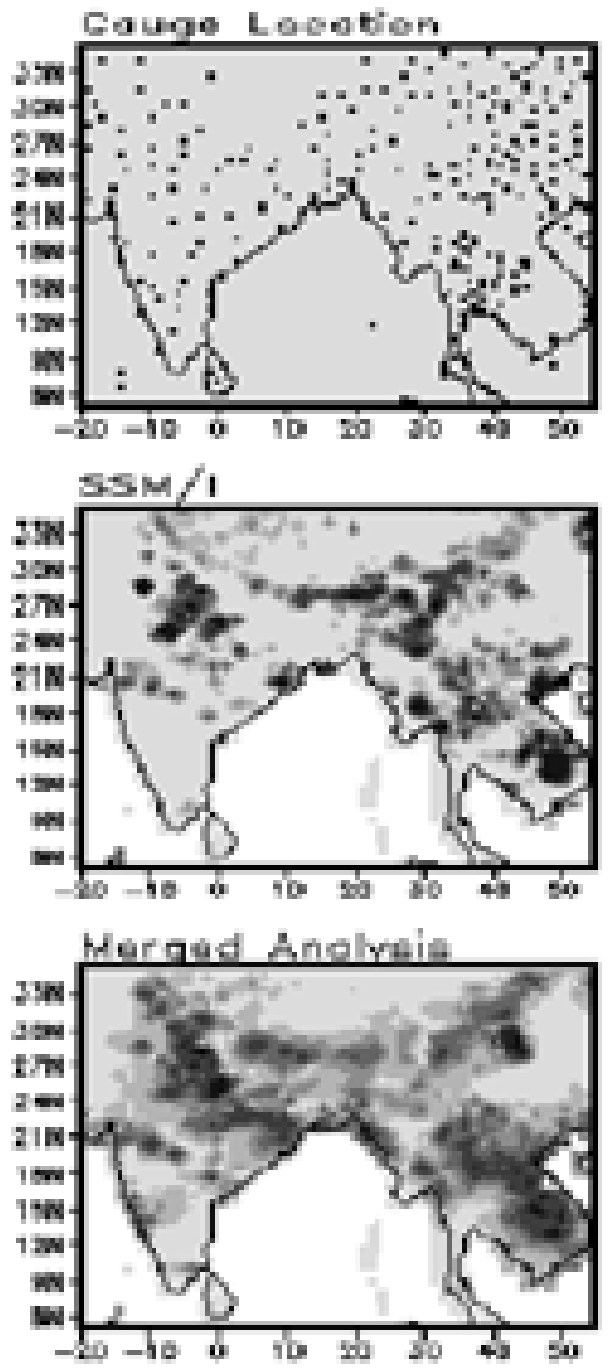

Figure 2. Daily Precipitation (Mm) For Jan, 1990, Obtained From GTS Gauge-Based Analysis, Satellite Estimates Of GPI, SSM/I And AMSU-B, and the Merged Analysis Along With GTS Gauge Stations 
Table 1. Output Bias and Correlation of Various Input Data Combinations

\begin{tabular}{ccc}
\hline Data & Bias (mm/day) & Correlation coefficient \\
\hline GPI only & 2.26 & 0.345 \\
SSM/I only & -0.24 & 0.321 \\
AMSU-A only & -0.15 & 0.095 \\
GTS+GPI+SSM /I+AMSU-A & -0.15 & 0.501 \\
GTS+GPI & -0.04 & 0.467 \\
\hline
\end{tabular}

The SPI calculation for any location is based on the long-term precipitation record that is fitted to a probability distribution, which is then transformed into a normal distribution so that the mean SPI for the location and desired period is zero. A drought event occurs any time; the SPI is continuously negative and reaches intensity of -1.0 or less (Table 1 ). The event ends when the SPI becomes positive. Each drought event, therefore, has a duration defined by its beginning and end, and intensity for each month that the event continues. The positive sum of the SPI for all the months within a drought event can be termed as the drought's "magnitude". Standardized Precipitation Index (SPI) is an index that was developed to quantify precipitation deficit at different time scales, and can also help assess drought severity (Das S, Choudhury MR, Nanda S., 2013).
$\mathrm{SPI}=\left\{\left(\mathbf{X}_{\mathrm{ij}}-\mathbf{X}_{\mathrm{im}}\right) / \sigma\right\}$

Where, $\mathrm{X}_{\mathrm{ij}}=$ is the seasonal precipitation

$\mathrm{X}_{\text {im }}$ is its long-term seasonal mean $\sigma$ is it's standard deviation.

SPI results computed from seasonal rainfall data were assigned to each grid cell of the study area, and reclassified based on drought severity classes. The value of drought generally varies from -2 to +2 . If the value is below 0 i.e between -1 to -2 then the probability of drought is more and it has adverse effect on crop productivity. If it remains below than obviously the productivity and health will be less. If the value is 0 than there would be no vegetation, and if the value is above 0 than vegetation will be high. (Table 2).

Table 2. The Standardized Precipitation Index (SPI) values and their description

\begin{tabular}{lll}
\hline SPI Value & Brief Description & Estimated Likelihood \\
\hline 2.00 and Greater & Extremely Wet & $\begin{array}{l}\text { about } 2.3 \% \text { of events (roughly 1 out of 40) are } \\
\text { expected to exceed 2.00 } \\
\text { about } 6.7 \% \text { of events (roughly 1 out of 15) are } \\
\text { expected to exceed 1.50 } \\
\text { about 16\% of events (roughly 1 out of 6) are } \\
\text { expected to exceed 1.00 } \\
\text { about } 68 \% \text { of values (roughly 2 out of 3) fall in } \\
\text { this range } \\
\text { about } 16 \% \text { of events (roughly 1 out of 6) are } \\
\text { expected to exceed -1.00 }\end{array}$ \\
\hline
\end{tabular}




\section{Results and Discussions}

\section{Rainfall Variability}

Rainfall variability map (Figure 3 ) is prepared from 1990-2014 in ArcGIS 9.3 and it is clearly visible that, the entire Rann of kutch and some other blocks are severely stressed by precipitation (12-97 $\mathrm{mm}$ yearly). The central part of Kutch is showing moderately stressed(337-375 mm yearly) and Rapar and Mundra is relatively less stressed (>375 mm yearly).

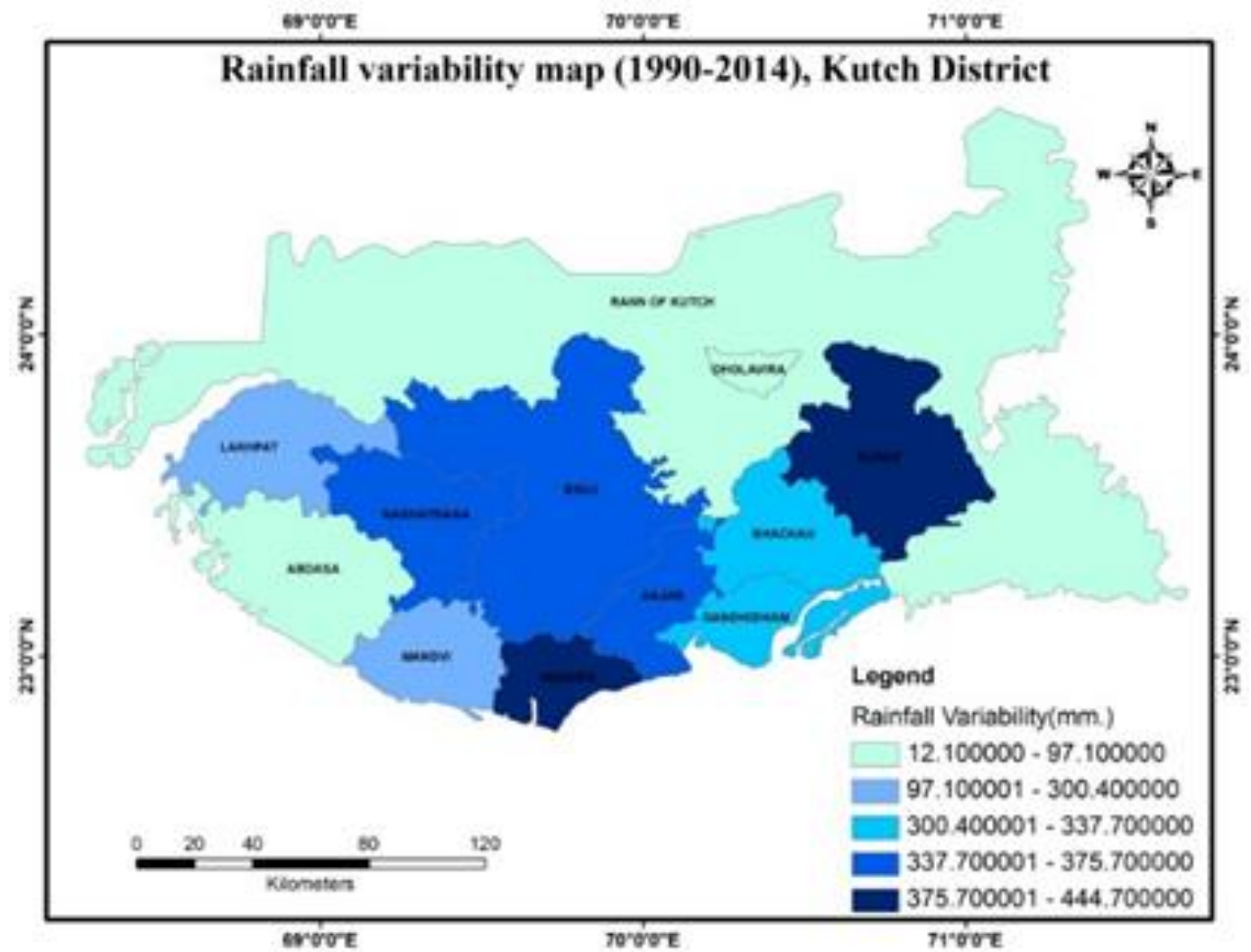

Figure 3. Rainfall variability map

\section{Land Use and Land Cover}

Land Use and Land cover map (Figure 4) represents the overall land use and land Cover classes area wise Using maximum likelihood classifier algorithm of ERDAS Imagine 9.3 software supervised classification has made over entire kutch district and the results significantly reveal that, Due to severe drought impacts, bare soil and barren land covers maximum area of kutch i.e., $20 \%$ and $24 \%$ respectively. Crop or cultivated land and vegetated land occupy small area. 


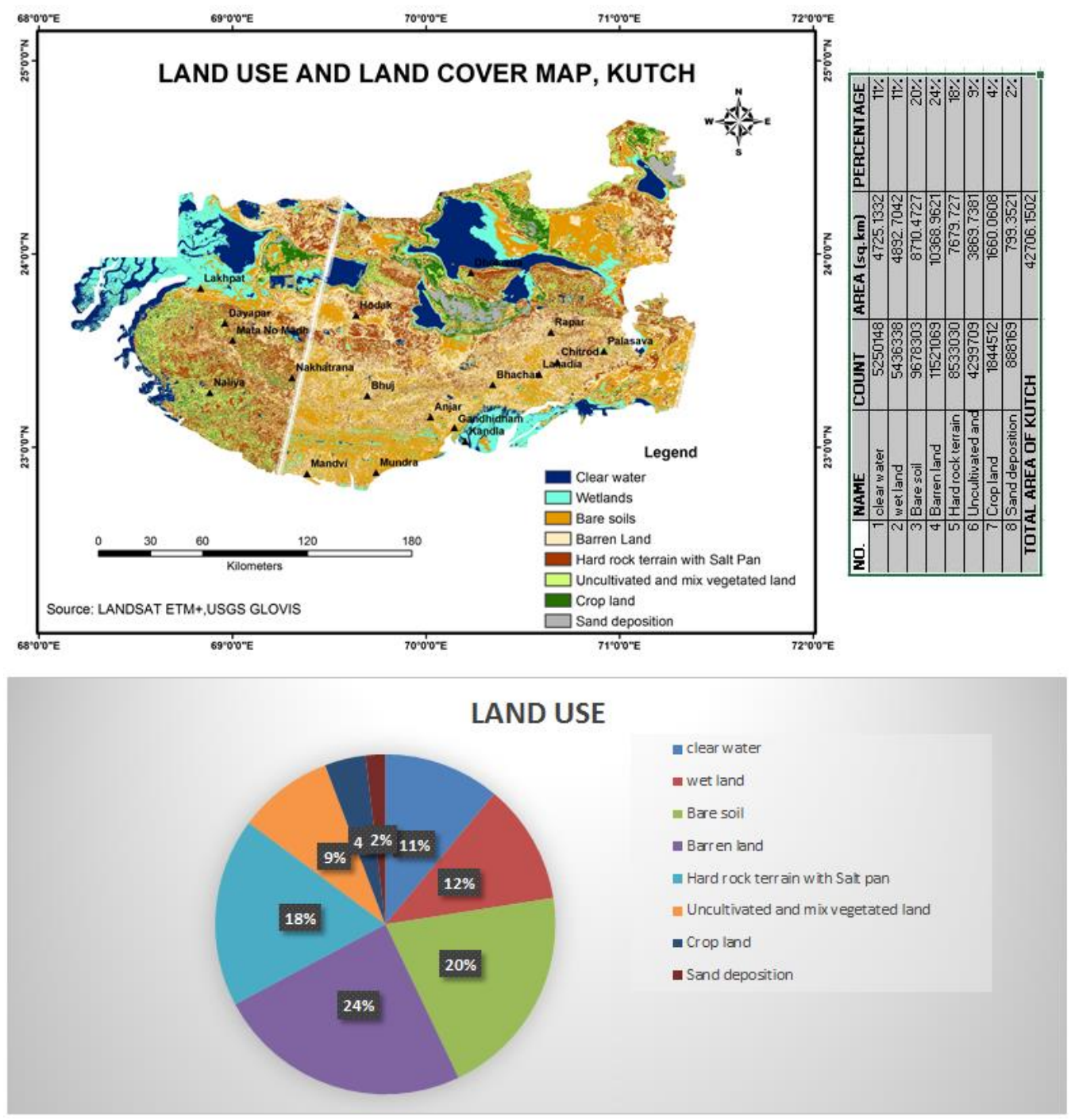

Figure 4. Land Use and Land cover classification map

\section{SPI based drought analysis and zonation}

SPI for individual blocks were computed from seasonal rainfall data were assigned to each grid cell of the study area, and reclassified based on drought severity (Figure 5\&6).
Similarly SPI for all other blocks of Kutch region have been calculated (see Annexure 1). 


\begin{tabular}{|c|c|c|c|c|c|c|c|c|c|c|c|c|c|c|c|c|c|c|c|c|c|c|c|c|c|c|c|}
\hline & & 홋 & 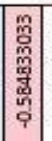 & 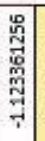 & 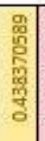 & 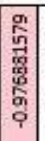 & 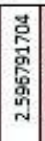 & 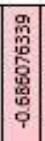 & 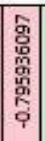 & 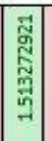 & 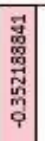 & 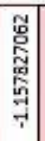 & 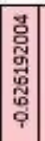 & 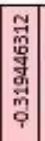 & 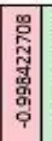 & 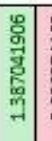 & & & 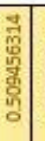 & 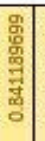 & 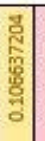 & 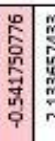 & & 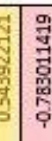 & 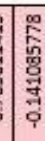 & 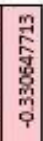 & \\
\hline & & 흔 & & & & & & & & & & & & & & & & & & & & & & & & & \\
\hline & & $\begin{array}{l}\text { 면 } \\
\frac{5}{3} \\
\frac{1}{4}\end{array}$ & & & & & & & & & & & & & & & & & & & & & & & & & \\
\hline & & 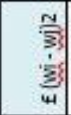 & 勇 & & & & & & & & & & & & & & & & & & & & & & & & \\
\hline & & 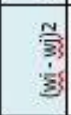 & 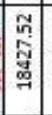 & $\begin{array}{l}\text { wa } \\
\vdots \\
0 \\
0 \\
0\end{array}$ & 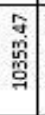 & 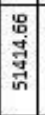 & 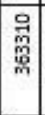 & 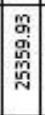 & 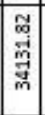 & 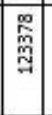 & 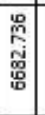 & 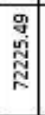 & \begin{tabular}{|l|}
\multirow{0}{0}{} \\
त्र \\
\end{tabular} & 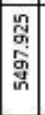 & \begin{tabular}{|c|} 
\\
0 \\
0 \\
0 \\
0 \\
0 \\
0
\end{tabular} & 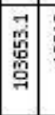 & 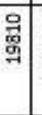 & 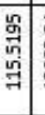 & 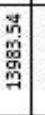 & 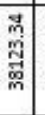 & 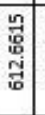 & 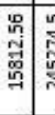 & 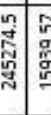 & 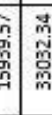 & 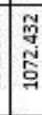 & 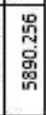 & zo \\
\hline & & \begin{tabular}{|l|}
3 \\
35 \\
3
\end{tabular} & 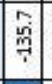 & 窎 & 恖 & \begin{tabular}{|l|}
\multirow{2}{*}{} \\
สุ่ \\
\end{tabular} & 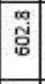 & 苗 & 总 & \begin{tabular}{|c|c|} 
\\
离 \\
\end{tabular} & \begin{tabular}{|l|l|} 
\\
\\
\\
\end{tabular} & 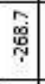 & 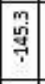 & 京 & ने & \begin{tabular}{|l|l|}
\multirow{2}{*}{} \\
\end{tabular} & 守 & î & 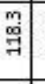 & \begin{tabular}{l|l}
0 \\
0 \\
0 \\
$g$
\end{tabular} & if & 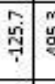 & 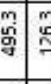 & 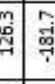 & స్త & 용 & $\begin{array}{l}\text { 틀 } \\
\text { 总 }\end{array}$ \\
\hline 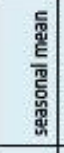 & 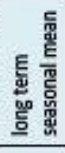 & & 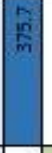 & & & & & & & & & & & & & & & & & & & & & & & & 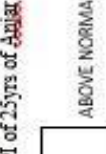 \\
\hline 愛 & $\circ$ & 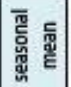 & 离 & ఏ్․ำ & $\stackrel{n}{E}$ & 章 & 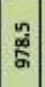 & 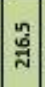 & 웜 & $\stackrel{\circ}{\AA}$ & 水 & 号 & ले & 号 & 姜 & 今. & 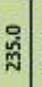 & 啇 & 章 & हี & రิ๊ & ఏें & : & 密 & 离 & & \\
\hline ğ & $z$ & & : & 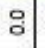 & : & : & 이 & 이 & 웅 & : & : & 8 & 8 & $:$ & $:$ & $:$ & : & : & : & $:$ & $:$ & $:$ & $:$ & 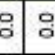 & : & : & 引 \\
\hline$\frac{3}{2}$ & $\Sigma$ & & : & : & 8 & 8 & : & : & : & \begin{tabular}{|l|}
8 \\
\end{tabular} & : & 8 & 8 & 8 & : & : & : & 8 & 용 & : & : & $:$ & $\begin{array}{l:}: \\
:\end{array}$ & $\begin{array}{l:}8 \\
\end{array}$ & : & : & 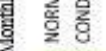 \\
\hline$\check{\Delta}$ & - & & : & : & : & : & : & 임 & 영 & : & 章 & 웅 & i & 임 & & : & : & & : & : & $:$ & : & $::$ & : : & 멱 & & \\
\hline : & $\simeq$ & & \begin{tabular}{|l|} 
㶽 \\
of
\end{tabular} & $\therefore$ & 品 & 8 & \begin{tabular}{|l|} 
学 \\
.
\end{tabular} & $\therefore$ & $\therefore$ & \begin{tabular}{|c|} 
查 \\
学
\end{tabular} & \begin{tabular}{|l|l|}
0 \\
N
\end{tabular} & $\begin{array}{l} \\
\text { ने }\end{array}$ & 옹 & 영 & & \begin{tabular}{|l|l|}
$\circ$ \\
\end{tabular} & \begin{tabular}{l|l} 
\\
\end{tabular} & 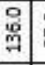 & \begin{tabular}{l|} 
\\
$\stackrel{2}{m}$
\end{tabular} & 곡 & 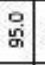 & $\begin{array}{lll} \\
\text { जi }\end{array}$ & 8 & \begin{tabular}{l|l} 
\\
\end{tabular} & : & 品 & \\
\hline$\frac{20}{4}$ & - & & \begin{tabular}{|l|l|} 
狊 \\
\end{tabular} & \begin{tabular}{l|l} 
\\
के
\end{tabular} & 염 & : & \begin{tabular}{|l|} 
\\
a \\
\end{tabular} & 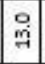 & $\begin{array}{l} \\
\end{array}$ & 竎 & 익 & जi & 㟔 & \begin{tabular}{|c|} 
\\
\end{tabular} & \begin{tabular}{|l|l|} 
& \\
\multirow{1}{*}{} & \\
\end{tabular} & 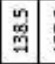 & 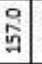 & \begin{tabular}{l|l}
0 \\
$\stackrel{9}{a}$
\end{tabular} & 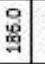 & 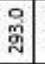 & 号 & 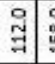 & : & \begin{tabular}{l|l}
\multirow{2}{*}{} & 0 \\
\end{tabular} & 역 & 영 & 岁 \\
\hline इ & - & & \begin{tabular}{|l|}
0 \\
0
\end{tabular} & 염 & 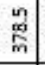 & 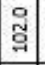 & \begin{tabular}{|l|l|} 
& \\
9 \\
0 \\
$m$
\end{tabular} & 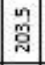 & \begin{tabular}{|l|} 
\\
$\stackrel{2}{*}$
\end{tabular} & 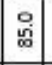 & 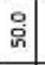 & $\begin{array}{ll} \\
\stackrel{9}{j} \\
\end{array}$ & \begin{tabular}{|l|} 
\\
解 \\
\end{tabular} & 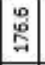 & 잉 & \begin{tabular}{|c|}
7 \\
勇
\end{tabular} & 율 & 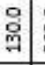 & 惫 & ฐ్ & घ․ & 齐 & 产索品 & $\begin{array}{ll}\text { ci } & \text { in } \\
\end{array}$ & 응 & 곡 & @ \\
\hline$\underline{\mathrm{g}}$ & $I$ & & : & $\therefore$ & gi & \begin{tabular}{|l|}
$\stackrel{0}{\theta}$ \\
$\stackrel{0}{*}$
\end{tabular} & \begin{tabular}{|l|} 
品 \\
ñ
\end{tabular} & $\therefore$ & 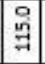 & \begin{tabular}{|c|}
\multirow{2}{*}{} \\
\end{tabular} & 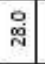 & $\begin{array}{ll} \\
\end{array}$ & 잉 & 辛 & \begin{tabular}{|l|l|} 
总 \\
in
\end{tabular} & \begin{tabular}{|l|} 
\\
\end{tabular} & \begin{tabular}{l|l} 
: \\
$: 5$
\end{tabular} & 임 & 임 & 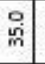 & 吕 & \begin{tabular}{l|l}
0 \\
$:$
\end{tabular} & ㄱำ & $: 8$ & 雍 & $\therefore$ & \\
\hline$\frac{7}{2}$ & 0 & & : & : & 임 & $:$ & : & $:$ & : & : & 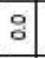 & : & : & : & : & : & : & $:$ & $:$ & $:$ & $:$ & : & \begin{tabular}{l|c}
$\circ$ & 5
\end{tabular} & $: 5$ & : & : & \\
\hline$\frac{a}{a}$ & 4 & & : & : & 8 & : & 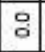 & 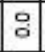 & $:$ & : & : & 8 & 8 & : & \begin{tabular}{|l|}
8 \\
\end{tabular} & $\therefore$ & : & 8 & : & $\therefore$ & $\therefore$ & $\therefore:$ & \begin{tabular}{c|c:}
$:$ & 0
\end{tabular} & $\begin{array}{c:}8 \\
8\end{array}$ & 8 & : & \\
\hline$\frac{5}{\Sigma}$ & $\omega$ & & : & $\therefore$ & : & : & : & : & : & : & : & : & : & : & : & $:$ & : & $\therefore$ & : & $\therefore$ & $\therefore$ & $:$ & $:$ & $:$ & : & : & 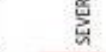 \\
\hline 원 & $\omega$ & & : & $:$ & $:$ & $:$ & 잉 & $\therefore$ & 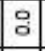 & : & $:$ & : & 8 & : & $:$ & $\therefore$ & : & $\therefore$ & $\therefore$ & $\therefore$ & $\therefore$ & $\therefore$ & $:$ & $8:$ & 8 & : & \\
\hline 든 & 0 & & : & : & : & \begin{tabular}{|l|} 
\\
0
\end{tabular} & $\begin{array}{l} \\
\end{array}$ & 웅 & : & : & \begin{tabular}{|l|l|} 
& \\
\end{tabular} & \begin{tabular}{|l|} 
\\
\end{tabular} & : & : & \begin{tabular}{|l|l|} 
& \\
\end{tabular} & \begin{tabular}{l|l} 
\\
\end{tabular} & 高 & \begin{tabular}{l|l} 
\\
$:$
\end{tabular} & : & : & : & \begin{tabular}{l|l}
$:$ & 5 \\
\end{tabular} & \begin{tabular}{l|l} 
& $\vdots$ \\
\end{tabular} & $\begin{array}{c:}: \\
\end{array}$ & $8:$ & : & \\
\hline 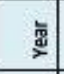 & 0 & & \begin{tabular}{|l|l|}
9 \\
\end{tabular} & 莺 & 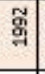 & $\begin{array}{ll}0 \\
0\end{array}$ & 甾 & 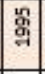 & 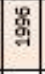 & \begin{tabular}{|l|} 
竎 \\
\end{tabular} & $\begin{array}{l}\text { : } \\
: \\
\end{array}$ & 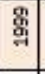 & \begin{tabular}{|l|} 
\\
\end{tabular} & 客 & 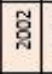 & 㖞 & : & 雚 & 蒿 & : & 塄 & \begin{tabular}{l|l} 
है। \\
\end{tabular} & व: & & ํㅠㅁ & 范 & \\
\hline 总惫 & $\infty$ & & s. & & & & & & & & & & & & & & & & & & & & & & & & \\
\hline $\begin{array}{ll}\frac{\mathrm{t}}{\mathrm{E}} \\
\frac{\mathrm{t}}{\mathrm{a}}\end{array}$ & 4 & $\begin{array}{l}\underline{5} \\
\frac{5}{y}\end{array}$ & & & & & & & & & & & & & & & & & & & & & & & & & \\
\hline
\end{tabular}

Figure 5. Standardized Precipitation Index (SPI) for Anjar Block, Kutch District 

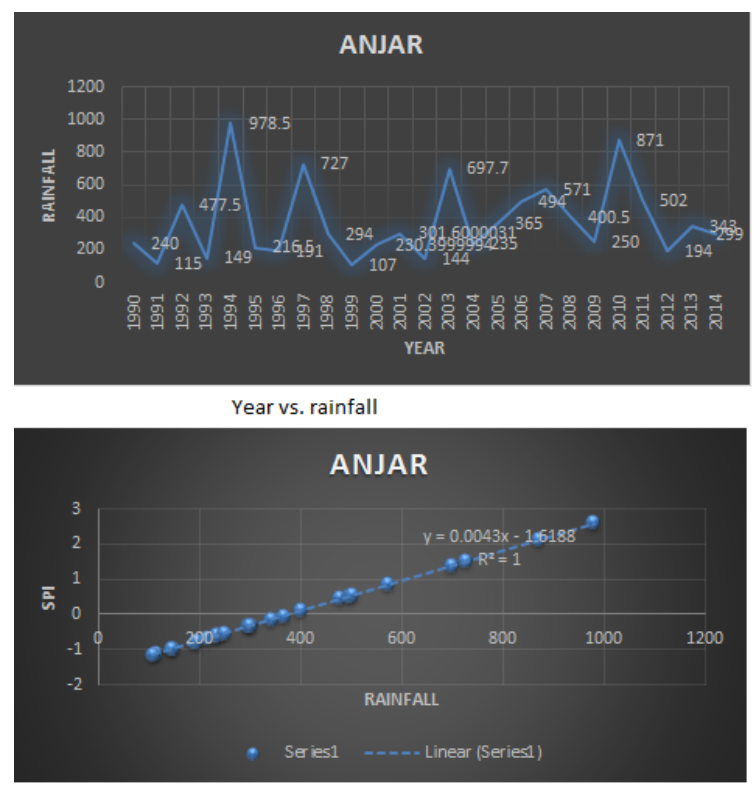

Correlation between rainfall and SPI

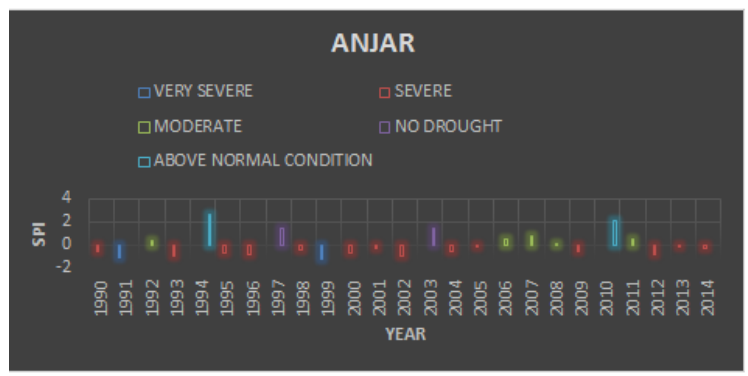

year vs. SPI

Figure 6. Graph Showing Yearly Variations of SPI of Anjar Block, Kutch District

\section{Conclusion and Recommendations}

The study demonstrated the SPI based approach for drought assessment through remote sensing techniques and seasonal long term rainfall data. It established a relationship between Rainfall and drought condition over agro-climatic regions of Kutch. Agriculture remains by far the most vulnerable and sensitive sector that is seriously affected by the impacts of climate variability and climate change, which is usually manifested through rainfall variability and drought. Occurrence of drought cannot be monitored by comparing the relative rainfall observed in various stations. To overcome these limitations, the use of SPI for drought monitoring was highlighted. It was concluded that SPI is an excellent means that gives indication of the drought characteristics like onset, severity, and spatial extent.
Through SPI, we can compare two different stations in different climatic regions regardless of the fact that they may have different normal rainfalls; because the rainfall is already normalized and compares the current rainfall with the average. The rainfall of two areas with different rainfall characteristics can be compared in terms of how badly they are experiencing drought conditions since the comparison is in terms of their normal rainfall. Hence, SPI is more efficient than other indices in spatial analysis of drought. From the SPI analysis it can be concluded that, Rann of Kutch, Abdasana, Rapar, Bhachau, Lakhpat are most drought prone areas.

\section{Acknowledgements}

Authors places on record their deep sense of gratitude to the Director, State water data centre 
and Indian Meteorological Department, Gandhinagar, Gujarat for providing the necessary data and information in this project. The authors are also thankful to the principal, Dr. Ami H. Shah of Smt. S. R. Patel Engg. College for providing research support. Besides, the authors wishes to express gratitude to the anonymous reviewers who help to improve the quality of this paper.

\section{References}

Das S, Choudhury MR, Nanda S. 2013. Geospatial Assessment of Agricultural Drought (A Case Study Of Bankura District, West Bengal), International Journal of Agricultural Science and Research (IJASR) ISSN 2250-0057 Vol. 3, Issue 1, Jun 2013, 1-28.

McKee T. B., Doesken N. J., Kleist J. 1995. Drought monitoring with multiple time scales. Reprints, 9th Conference on Applied Climatology, January 15-20, Dallas, Texas, 233-236.

McKee T. B., Doesken N. J., Kleist J. 1993. The relationship of Drought frequency and duration to time scales. Preprints, 8th Conference on Applied Climatology, January 17-22, Anaheim California, 179184.

Mishra A. K., Desai V. R. 2005. Spatial and temporal drought analysis in the Kansabati river basin, India. International River Basin Management, 3(1), 31-41.

Nalbantis I., Tsakiris G. 2009. Assessment of hydrological drought revisited. Water Resources Management, 23, 881-897.

Palmer W. C. 1965. Meteorological Drought. U. S. Weather Bureau, Washington DC, Research Paper No. 45.

Palmer W. C. 1968. Keeping track of crop moisture conditions, nationwide: the new Crop Moisture Index. Weatherwise, 21, 156-161.

Rawat K.S., Tripathi V.K., George Paul., Mishra A.K. 2014. The Standard Precipitation Index Analysis for Drought Assessment in Indian Agro-Climate Regions, using of GIS and Remote Sensing Data, NCWES - 2014, 223-232.

Smakhtin V. U., Hughes D. A. 2004. Review, Automated Estimation and Analyses of Drought Indices in South Asia. International
Water Management Institute, Colombo, Sri Lanka, Working Paper No. 83.

Tsakiris G., Pangalou D. 2007. Vangelis H., Regional draught assessment based on the Reconnaissance Draught Index (RDI). Water Resources Management, 21, 821 833.

Wilhite D., Glantz M. R. 1987. Understanding the drought phenomenon - The role of definitions, in Wilhite, David, Easterling, William, and Wood, David, eds., Planning for drought: Boulder, Colo., Westview Press, 11-27.

Wilhite D. A., Hayes M. J., Svoboda M. D. 2000. Drought monitoring and assessment: status and trends in the United States. In: Vogt J. V., Somma F. (eds) Drought and drought mitigation in Europe. Kluwer Academic, Dordrecht, 149-160. 


\section{Annexure: 1}

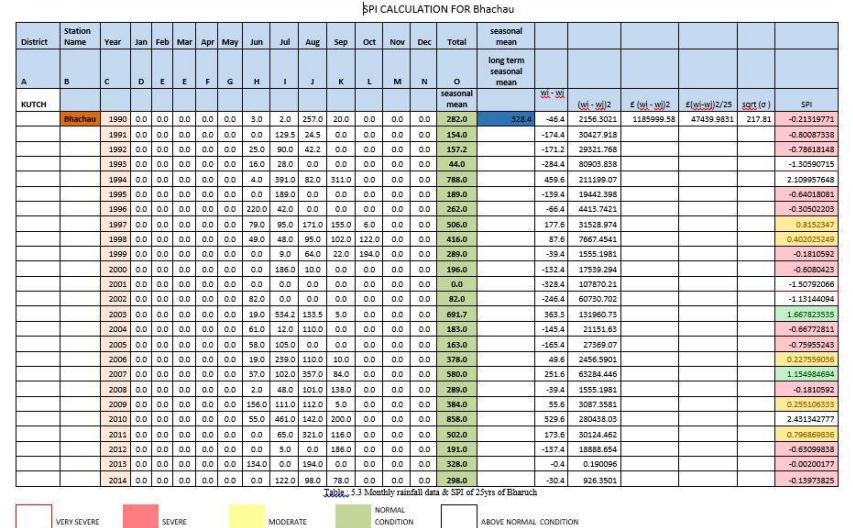

(A)

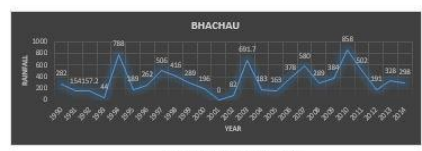

Graphi 5.4 Year 3s Rainfall

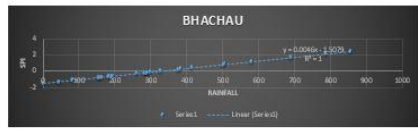

Graph: 5.5 Regression betwreen rainfall and SPI

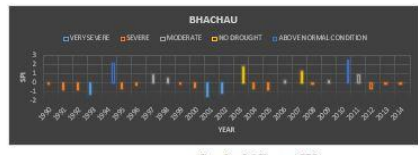

Gcaph $<5.6$ Year wa SPI

(B)

Figure 7. (A) SPI Calculations for BHACHAU

(B) Graph Shoving Yearly Variations of SPI

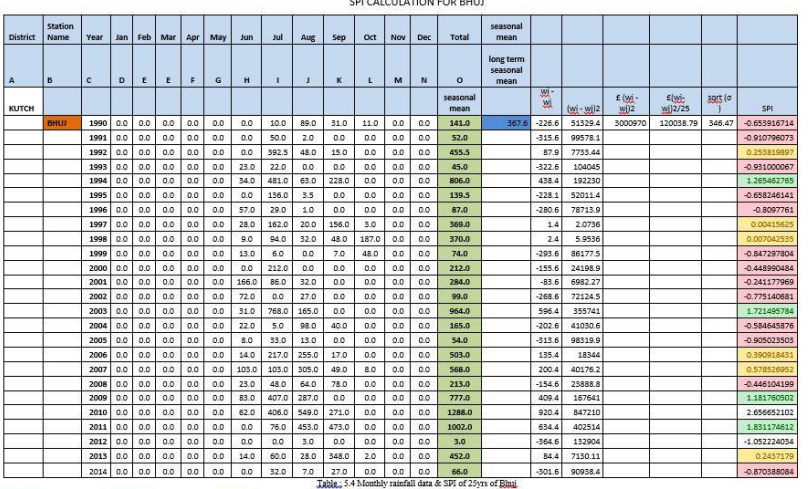

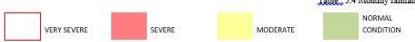

(A)

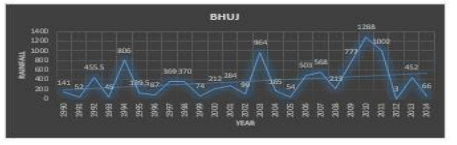

Graplei 6.7Year ya Rainfal

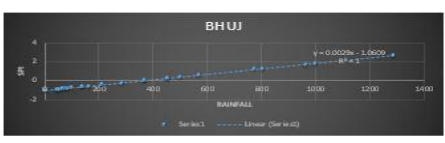

Scraph: 5.8 Regression between rainfall and SPI

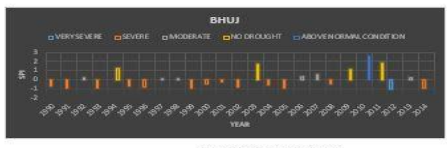

Gxaphi 6.9 Year wa SPI

(B)

Figure 8. (A)SPI Calculations for BHUJ

(B) Graph Shoving Yearly Variations of SPI

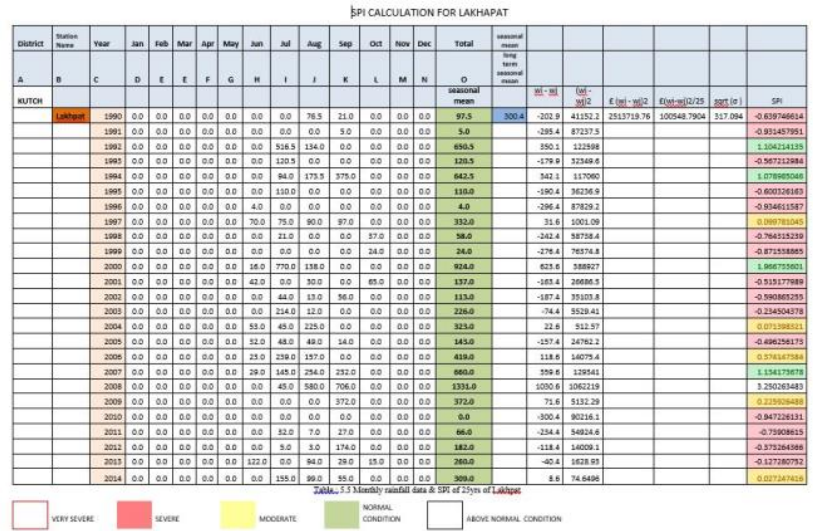

(A)

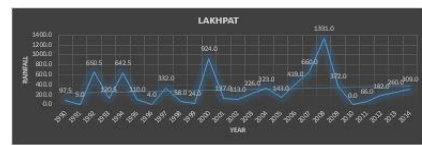

Graphi. 5.10 Year ra Raintall

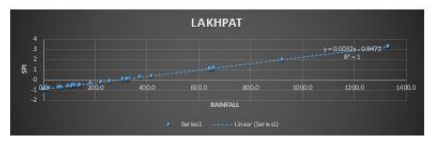

Graph: 5.11 Regression betrieen rainfall and SPI

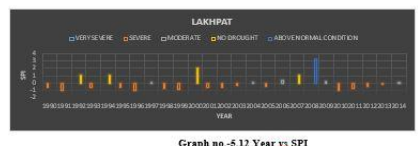

(B)

Figure 9. (A)SPI Calculations for LAKHAPAT

(B) Graph Shoving Yearly Variations of SPI 


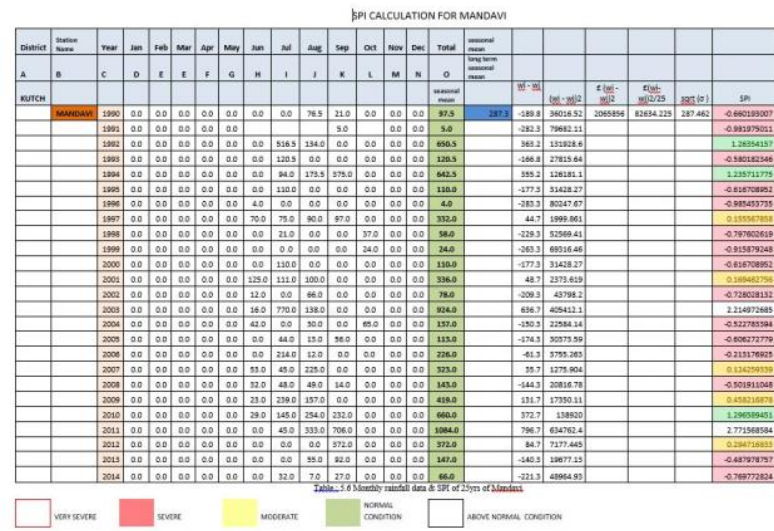

(B)
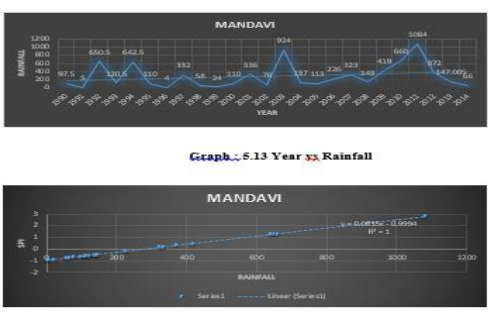

Gcapli: 5.14 Regression between rainfall and SPI

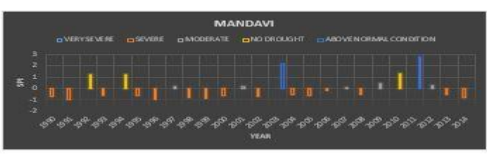

Gxaph: 5.15 Year xa SPI

(B)
Figure 10. (A)SPI Calculations for MANDVI

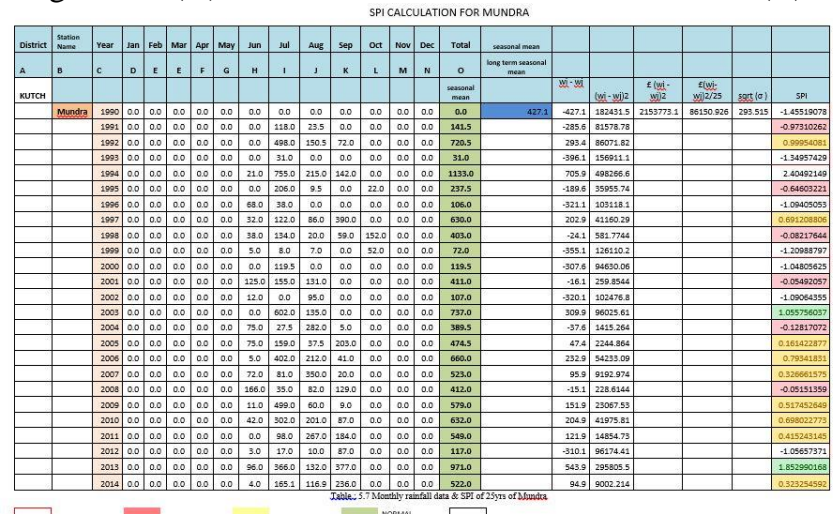

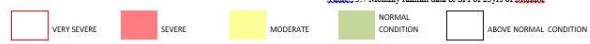

(B)
(B) Graph Shoving Yearly Variations of SPI

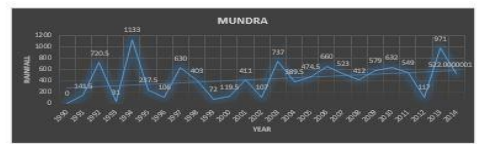

Graph 5.16 Year 55 Rainfall
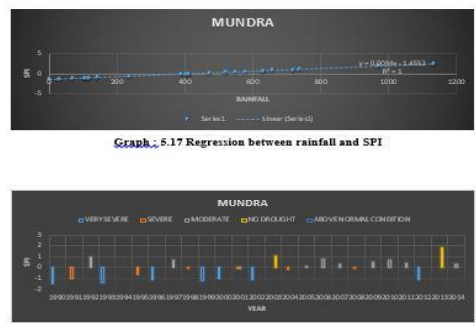

Graphi 6.18 Year $y x S P$

Figure 11. (A)SPI Calculations for MUNDRA

(B) Graph Shoving Yearly Variations of SPI

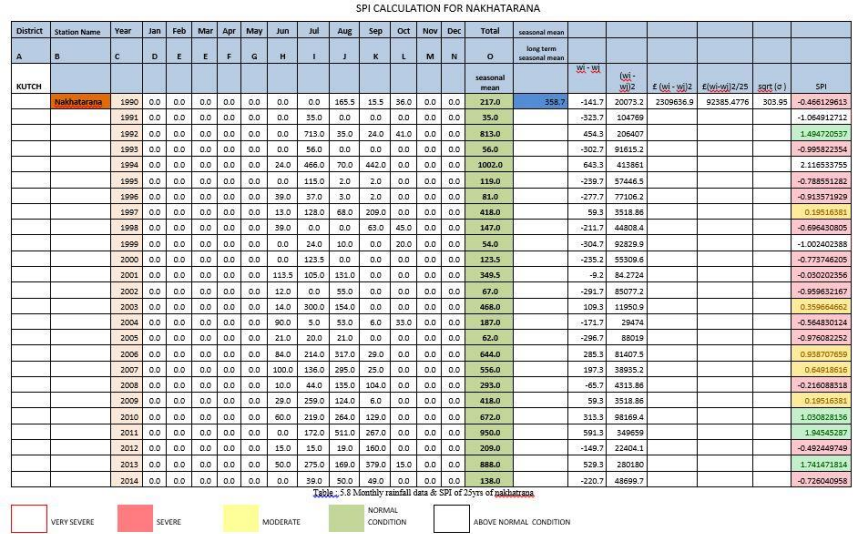

(B)

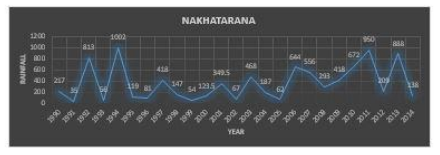

Sraphi 5.19 Year y, Rainfall
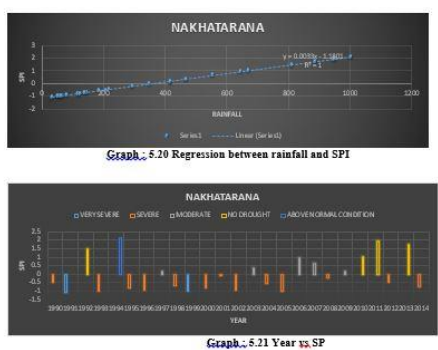

(B)

Figure 12. (A)SPI Calculations for NAKHATRANA $\quad$ (B) Graph Shoving Yearly Variations of SPI 


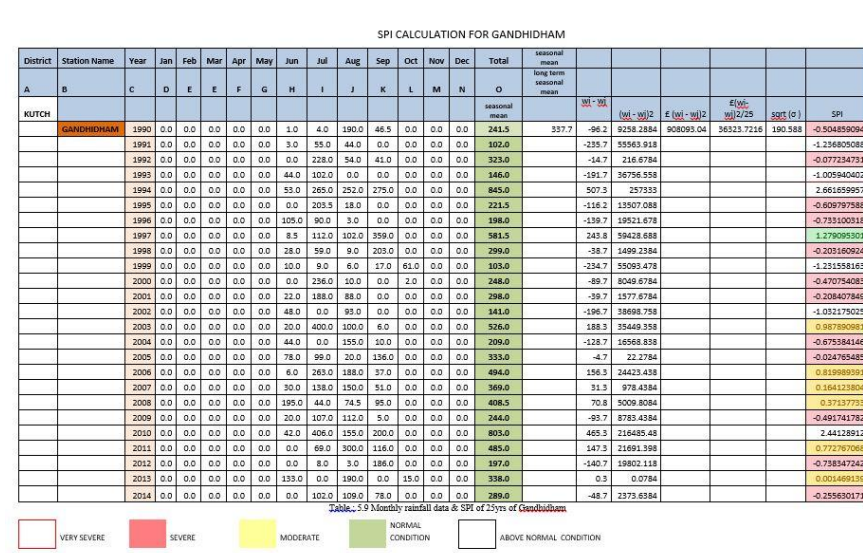

(C)

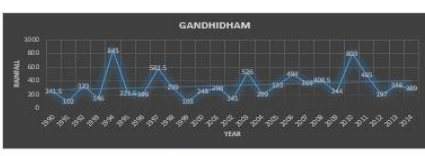

Graph: 6.22 Year rs Rainfall
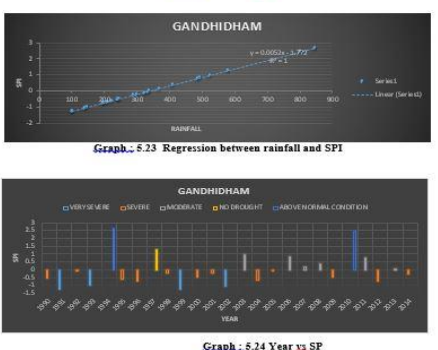

(B)

Figure 13 (A)SPI Calculations for GANDHIDHAM $\quad$ (B) Graph Shoving Yearly Variations of SPI

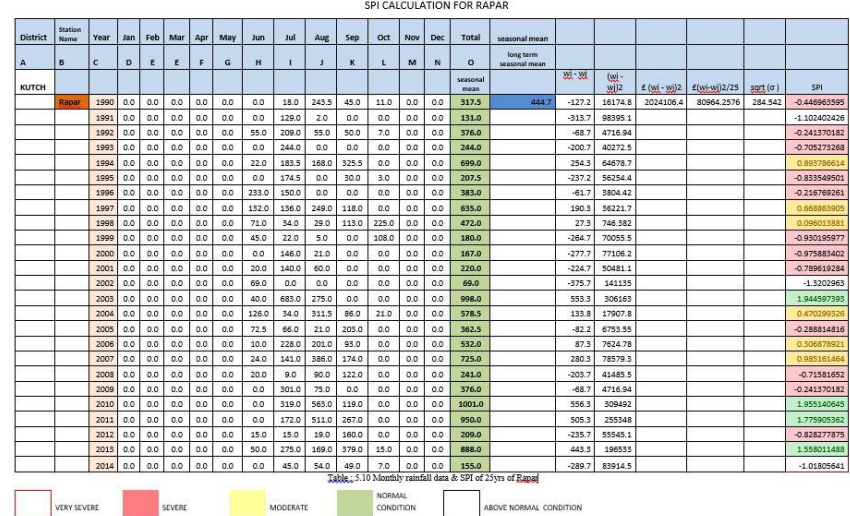

(C)

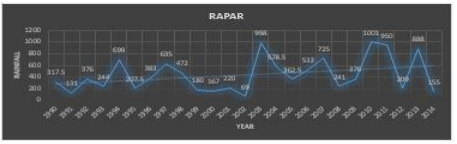

Geraph: 5.25 Year zs Rainfall

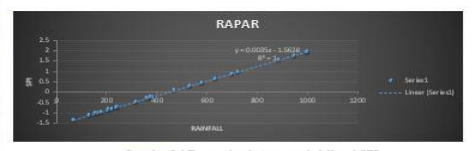

Graphi: 5.6 Regression betwreen rainfall and SPI

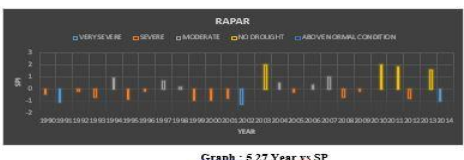

(B)

(B) Graph Shoving Yearly Variations of SPI

Figure14. (A)SPI Calculations for RAPAR

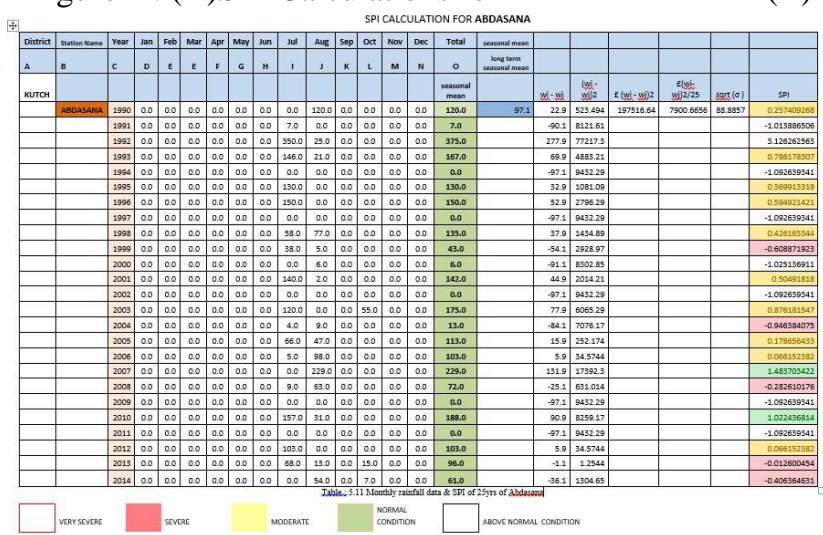

(C)

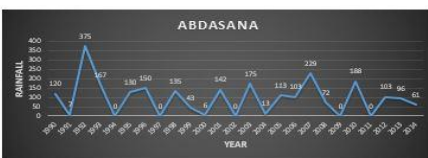

Graph ; 5.28 Year ys Rainfall
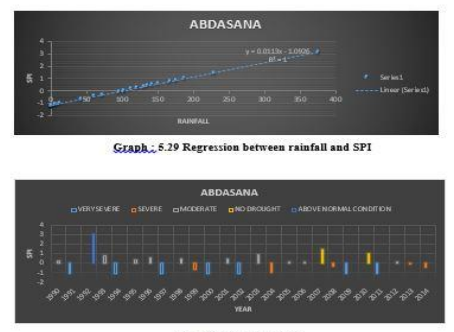

Craph 5.30 Year $125 P$

(B)

Figure 15.(A)SPI Calculations for ABDASANA

(B) Graph Shoving Yearly Variations of SPI 


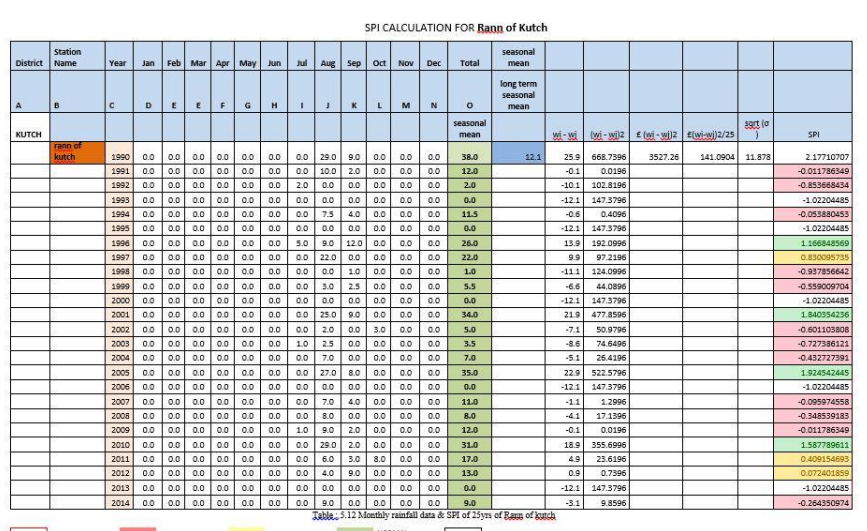

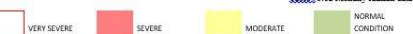

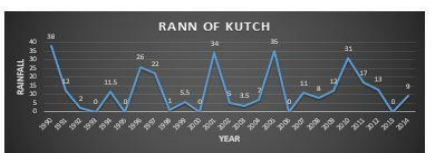

Graphi 5.31 Year xs Rainfall

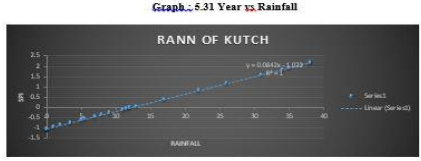

Craphi:5.32 Regression between rainfall and SPI

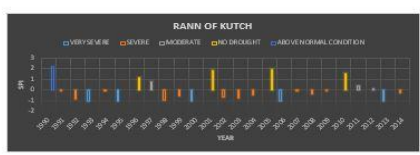

Geraphi 5.33 Year sa SPI

(D)

(B)

Figure 16.(A)SPI Calculations for RANN OF KUTCH (B) Graph Shoving Yearly Variations of SPI 